\title{
Correction of Unilateral Nostril Hypoplasia with Z-Plasty in a Child
}

Dong-Han Lee, Kap Sung Oh

Department of Plastic Surgery, Samsung Medical Center, Sungkyunkwan University School of Medicine, Seoul, Korea

No potential conflict of interest relevant to this article was reported.

\begin{abstract}
Unilateral nostril hypoplasia is an extremely rare congenital malformation of unknown etiology, and only a few cases have been reported in literature. Owing to variability and complexity of the deformity, surgical correction of unilateral nostril hypoplasia represents one of the most significant reconstructive challenges to reconstructive plastic surgeons. We report a 7-year-old Vietnamese child with nasal and periocular deformity resembling a craniofacial cleft. Grossly, the right nostril was patent but with alar rim deformity, and the left nostril was not readily identifiable. A dystopic medial canthus was present on the left side as well. Closer inspection and palpation of the left side of nose revealed a patency through the soft tissue and underlying bony structure, Thus, a new alar rim were reconstructed with an irregularly shaped Z-plasty to create patency on the involved side. Simulatneously, a second Z-plasty was performed to address the medial canthal deformity. Postoperative appearance and function was sastisfactory at one-year follow up visit. In the treatment of patients with nostril hypoplasia, a careful preoperative physical examination is a prerequisite, and Z-plasty can be a valuable option for surgical correction.
\end{abstract}

Keywords: Nose / Congenital / Malformation / Z-plasty

\section{INTRODUCTION}

Unilateral nostril hypoplasia is an extremely rare congenital malformation that may affect the soft tissue, cartilaginous, and/or bony structures of the nose. Manifestations can vary widely, with severe cases involving total obliteration of soft tissue and malformation or cleft of underlying structures including premaxilla and choanae. This deformity is very rare, but can occasionally be found in atypical cases with cerebro-oculo-nasal syndrome, interhemispheric fusion, or craniofacial cleft [1,2]. Because of this, surgical techniques for correction of this deformity have been limited to few case reports, which include Z-plasty, local flaps, cheek rotation flap, rotation and advancement flap, and tissue ex-

\section{Correspondence: Kap Sung Oh}

Department of Plastic Surgery, Samsung Medical Center, Sungkyunkwan University School of Medicine, 81 Irwon-ro, Gangnam-gu, Seoul 135-710, Korea E-mail: kapsung.oh@samsung.com

Received June 29, 2014 / Revised July 23, 2014 / Accepted July 29, 2014 pansion [3-6]. We report a case of unilateral nostril hypoplasia in a patient whose nasal obstructive symptom was dramatically improved by Z-plasty.

\section{CASE REPORT}

A 7-year-old Vietnamese girl presented with left-sided nostril hypoplasia at Lao Cai Hospital in Vietnam while senior author was providing volunteer medical services (Fig. 1). The guardians were unable to give any history relevant to family history or hereditary syndromes. The child had inferiorly displaced medial canthus and lower eyelid coloboma, which made her left side of the midface appear shorter than the right side, as well as a superiorly displaced alar base and deformed alar rim (Fig. 1). She suffered from soreness and dryness in the left eye from the periocular deformities. The alar rim deformity was sufficiently severe to make breathing through the left nostril virtually impossible, and to 
cause the patient to appear as if she had a single nostril. During a second examination on the day of preoperative planning, a pinpoint opening was identified in the soft-tissue, and the patency was found to extend through the bony structure underlying the affected alar rim, which became a key functional indication for a construction of a new nostril.

The nasal and eyelid deformities were addressed by a sequence of Z-plasties. For repositioning the eyelid, Z-plasty was designed for the medial canthal area, and a discrete irregular Z-plasty was separately designed for the alar area (Fig. 2). Flaps and underlying facial expression muscles were carefully dissected. The superiorlybased nasofrontal flap was advanced and turned over to reconstruct the nasal ala, and a laterally-based cheek flap was advanced

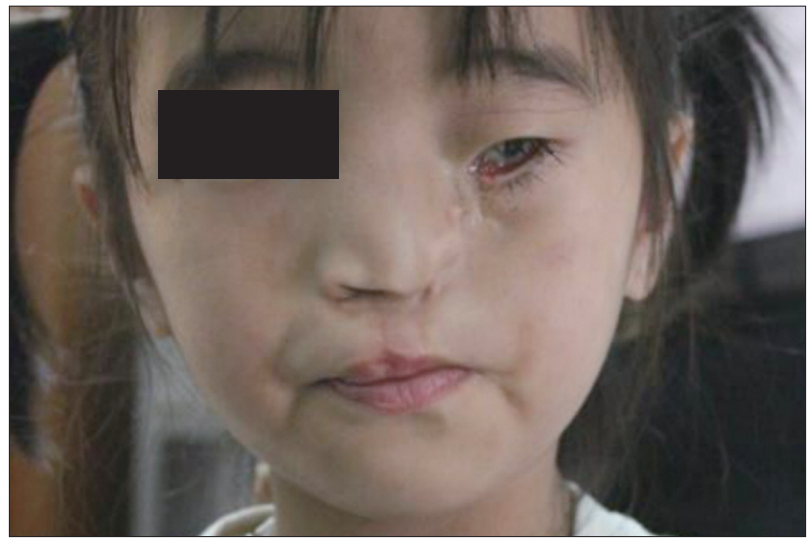

Fig. 1. Preoperative photograph of the patient. The left medial canthus was inferiorly displaced with obvious lower eyelid coloboma. The left nasal ala was superiorly displaced with a highly restrictive soft-tissue opening, which was appreciated only after a repeat exam. supero-medially to lengthen the perinasal midfacial area. At the same time, Z-flaps designed on medial canthal area were transposed to reposition the left medial canthus and to conceal the lower eyelid coloboma. Intraoperatively, nasal turbinates were slightly hypoplastic and choanal space was relatively well maintained on the affected side. Thus, sufficient space was obtained in the left nostril after transposing the Z-flaps on the left nasal ala. After confirming adequate passage of Nelaton catheter through the newly formed left nostril, transalar suture using absorbable stitch was performed on left ala to maintain the dimension of the opening. Immediately after the operation, wet gauze was packed to maintain the contour of newly-constructed left nostril (Fig. 3). The skin flaps survived without any complications, and the packing was removed

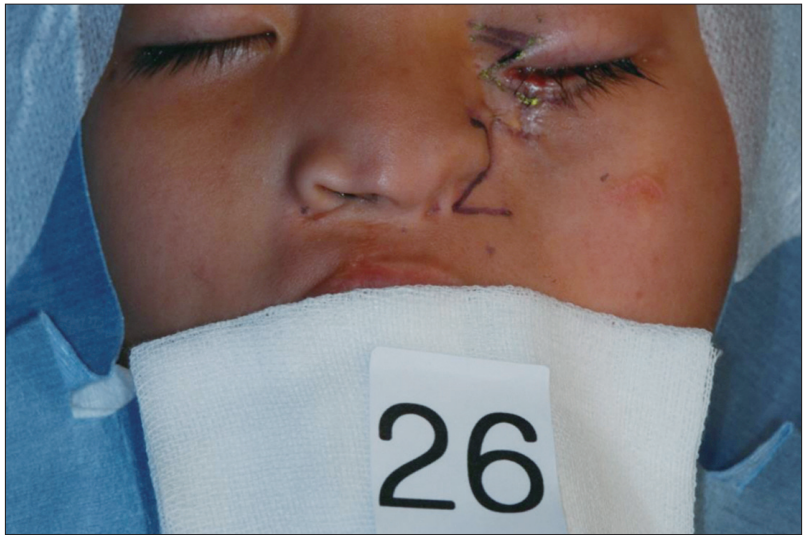

Fig. 2. Intraoperative design of Z-plasties. The upper Z-plasty was intended to elevate the medial canthal segment to its orthotopic position. The lower irregular Z-plasty was intended to transpose the alar base to its normal position and to construct a left nostril.
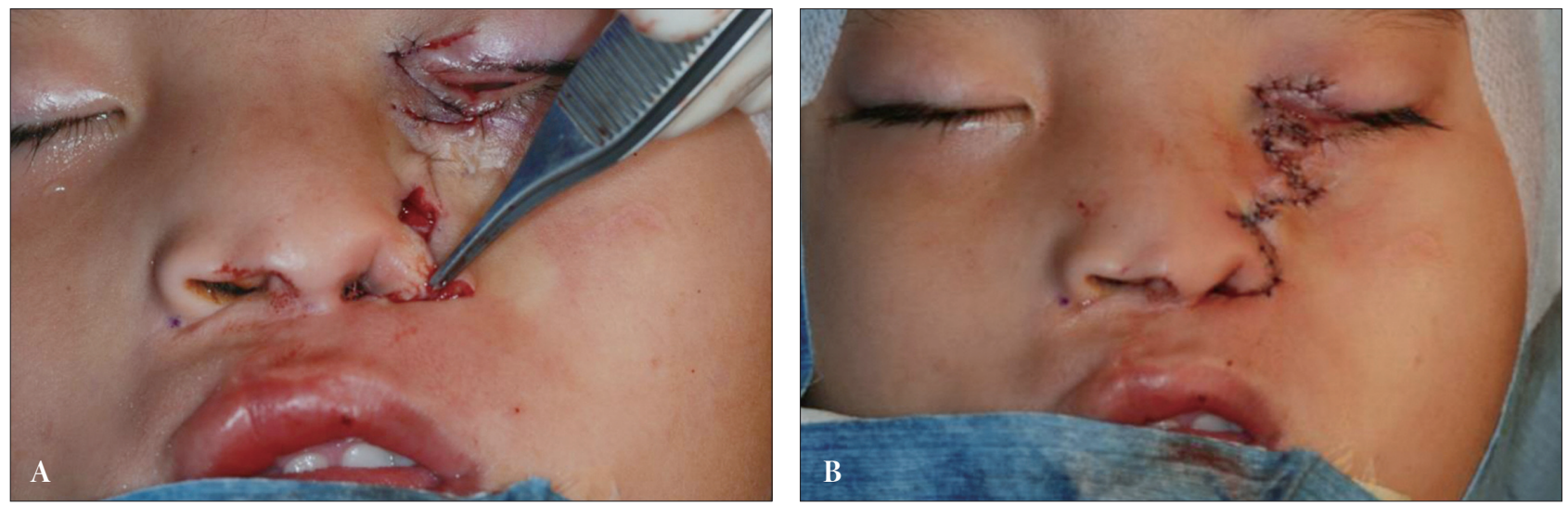

Fig. 3. (A) Lower Z flaps were transposed to correct the patient's nasal deformity and to improve breathing discomfort. (B) Immediately after the surgery, the medial canthus and alar base were moved to nearly symmetrical positions. 
on postoperative day 3 . The patient was very pleased to find that breathing through the nose required much less effort. The senior author completed his volunteer services in Vietnam and returned to Korea several days after this. Subsequently, another medical team visited Vietnam for volunteer services and was able to followup with the patient (Fig. 4). The patient had recovered well after surgery and was satisfied with the results. The reconstructed eyelid and nostril were favorably maintained.

\section{DISCUSSION}

Nostril hypoplasia is an extremely rare congenital anomaly and may be associated with severe congenital syndromes. In this case, the underlying structures were scarcely affected, while soft tissue opening was nearly absent. Similar phenotypic appearances are also found in patients with craniofacial cleft [1,2]. There are several reports of patients with cerebro-oculo-nasal syndrome with abnormal nares, and Richieri-Costa and Ribeiro $[7,8]$ had reported a case of atypical interhemispheric fusion with a functional single nostril [9]. However, the general facial appearances in those cases were different from the facial appearance of the patient presented here. On the other hand, craniofacial cleft patients have facial appearances similar to the preoperative facial appearance of this case.

The most widely accepted and easily applicable classification for facial cleft was proposed by Tessier [10] in 1976. The Tessier number 3 cleft is one of the most challenging malformations to reconstruct and is characterized by inferior displacement of the

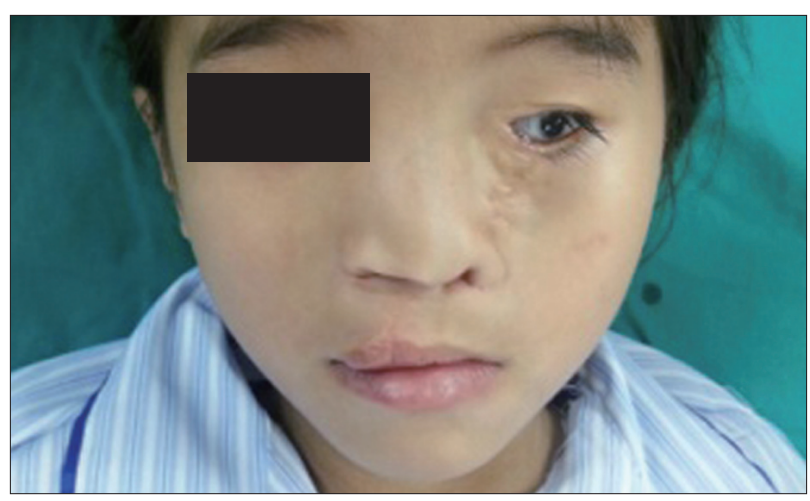

Fig. 4. Photograph at one year after surgery. The medial canthal location was stable, and the left nostril remained patent. medial canthus, superior displacement of the alar base, cleft lip and palate, coloboma of lower eyelids, nasolacrimal abnormality, cleft of the inferomedial orbital wall, and teloorbitism [11]. The Tessier number 4 cleft has been reported in less than 50 cases in the literature and is characterized by laterally and inferiorly displaced canthus, upward rotated nasal ala, and cleft lip lateral to the Cupid's bow [12]. Based on previous reports, the present case appears to represent either a number 3 or $4 \mathrm{cleft}$ on the clinical spectrum of Tessier clefts. To the best of our knowledge, this is the first report on the application of multiple Z-plasties in successful correction of facial cleft patient in Korea. Because of the rarity, surgical techniques regarding single nostrils are based on case reports, which include local flaps, cheek rotation flap, and tissue expansion. Z-plasty may be a simple but powerful tool to repair the key elements in most cases of nostril hypoplasia or Tessier number 3 cleft $[1,13,14]$. It can be used to correct ectropion, which may be a secondary consequence of a vertical loss of both the anterior and posterior lamellae of lower eyelids [13]. Also, Z-plasties can be chosen to repair the three main components of Tessier cleft 3 - the lower eyelid ectropion, the cleft lip, and the cleft between nose and malar area $[1,14]$. In our case, we designed two asymmetric Zflaps, one containing the nasal alar segment and another on the nostril base. By transposing each flap, the abnormal nasal ala was rearranged to restore symmetry in the midface, while successfully constructing a nostril. The discrete Z-plasty on left medial canthal area contributed to elongation of the left midface.

In conclusion, the fundamental principle in correcting single nostril is a precise diagnosis, including genetic analysis and the identification of accompanying anomalies, followed by thorough examination of the nasal components before detailed surgical planning. Because of the wide variation in anatomic configurations, the correction of single nostril should be individualized to achieve optimal results. The present case shows that Z-plasty may be a simple and powerful tool in cases of single nostril.

\section{REFERENCES}

1. Cizmeci O, Kuvat SV. Tessier no. 3 incomplete cleft reconstruction with alar transposition and irregular z-plasty. Plast Surg Int 2011; 
2011:596569.

2. Gawrych E, Janiszewska-Olszowska J, Chojnacka H. Tessier type 3 oblique facial cleft with a contralateral complete cleft lip and palate. Int J Oral Maxillofac Surg 2010;39:1133-6.

3. Longaker MT, Lipshutz GS, Kawamoto HK Jr. Reconstruction of Tessier no. 4 clefts revisited. Plast Reconstr Surg 1997;99:1501-7.

4. Stricker M. Craniofacial malformations. Edinburgh; New York: Churchill Livingstone; 1990.

5. Giglio A, Ruschel FF, Barcellos C, Pavelecini M, Chem RC. Rotation and advancement flap of the cheek in the treatment of rare craniofacial clefts. J Craniofac Surg 2008;19:1411-5.

6. Menard RM, Moore MH, David DJ. Tissue expansion in the reconstruction of Tessier craniofacial clefts: a series of 17 patients. Plast Reconstr Surg 1999;103:779-86.

7. Richieri-Costa A, Ribeiro LA. Cerebro-oculo-nasal syndrome, a disorder with some manifestations suggestive of the holoprosencephalic spectrum: new case and imaging review of previous cases. Am J Med Genet A 2005;136:352-3.

8. Ribeiro LA, Richieri-Costa A. Atypical interhemispheric fusion with a cebocephalic-like functional single nostril nose and a novel SHH mutation. Am J Med Genet A 2005;136:348-9.

9. Guion-Almeida ML, Zechi-Ceide RM, Richieri-Costa A. Cerebrooculo-nasal syndrome: 13 new Brazilian cases. Am J Med Genet A 2007;143:3252-66.

10. Tessier P. Anatomical classification facial, cranio-facial and latero-facial clefts. J Maxillofac Surg 1976;4:69-92.

11. Wenbin Z, Hanjiang W, Xiaoli C, Zhonglin L. Tessier 3 cleft with clinical anophthalmia: two case reports and a review of the literature. Cleft Palate Craniofac J 2007;44:102-5

12. Sari A, Yavuzer R, Ozmen S, Tuncer S, Latifoglu O. Early bone grafting in Tessier number 4 cleft: a case report. J Craniofac Surg 2003;14: 406-10.

13. Uemura T, Onizuka T, Suse T, Saiga A, Matsumoto N, Yoshimaru C, Nakamura H, Mitsukawa N. Composite Z plasty for cicatricial ectropion of Tessier III cleft. J Craniofac Surg 2004;15:51-3.

14. Mishra RK, Purwar R. Formatting the surgical management of Tessier cleft types 3 and 4. Indian J Plast Surg 2009;42 Suppl:S174-83. 